\title{
Feasibility and Safety of Laparoscopic Total Mesometrial Resection in Early-stage Cervical Cancer
}

\author{
SARA NASSER, JUMANA ALMUHEIMID, HELMUT PLETT, JALID SEHOULI and MUSTAFA ZELAL MUALLEM \\ Department of Gynecology, Campus Virchow-Klinikum, Charité Medical University, Berlin, Germany
}

\begin{abstract}
Aim: In this study we aimed to analyze the safety and feasibility of total mesometrial resection (TMMR) using the laparoscopic approach. Patients and Methods: Laparoscopic TMMR and pelvic lymphadenectomy (LNE) was carried out in 34 patients with cervical cancer FIGO IA-IIB from April 2012-April 2016 at our tertiary center. Para-aortic LNE was performed when indicated. The main outcomes included surgical margins, a number of retrieved lymph node, intra- and post-operative complications, and recurrence rates. Results: Complete microscopic tumor resection was confirmed in 33/34 (97\%) patients. No conversion to open surgery was necessary. Mean intraoperative blood loss was only $65.2 \mathrm{ml}$ with no blood transfusions required. Intra-operative complications occurred in $4 / 34(11.8 \%$ ) cases (2 bladder injuries and 2 ureteric injuries). Post-operative complications were observed in 10/35 (29.4\%) cases. Only one complication occurred after 30 days (vesico-vaginal fistula). There was a loco-regional recurrence within a mean follow-up time of 25.9 months. Conclusion: Laparoscopic TMMR appears to be feasible and safe in the treatment of early-stage cervical cancer. Further large-scale studies are required.
\end{abstract}

Cervical cancer continues to be a serious health issue worldwide. It is the second most commonly diagnosed cancer and third leading cause of cancer death among females in less developed countries. There were an estimated 527,600 new cervical cancer cases and 265,700 deaths worldwide in 2012 (1). Radical hysterectomy is the current gold-standard surgical treatment for cervical cancer. There have also been

Correspondence to: Dr. med. Dr. (syr.) Mustafa Zelal Muallem Charité Universitätsmedizin Berlin, Campus Virchow-Klinikum, Augustenburger Platz 1, 13353 Berlin, Germany. Tel: +49 30450664373, Fax: +49 30450564900, e-mail: MustafaZelal.Muallem@charite.de

Key Words: TMMR, laparoscopic, cervical cancer. several attempts to standardize the technique of radical hysterectomy required for different tumor extension, with variable outcomes (2). In 2009, Höckel et al. proposed total mesometrial resection (TMMR), developed by open surgery, as an alternative to radical hysterectomy for cervical cancer of stages IB-IIA and selected cases of IIB (3).

TMMR is based on the ontogenic compartment theory, which states that malignant tumor growth is confined to permissive compartments derived from a common primordium in embryonic development (2). It completely removes the Müllerian compartment except for the distal parts, where the resection is performed intracompartmentally to preserve a functional vaginal vault (3). Current evidence shows that TMMR can be standardized for all patients with locally defined tumors and appears to reduce morbidity, improve outcome and can potentially eliminate the need for adjuvant chemotherapy (2-5). In a prospective study of 212 consecutive patients who underwent TMMR without adjuvant radiation, Höckel et al. concluded that the resection of the embryologically defined uterovaginal compartment seems to be pivotal for pelvic control in patients with cervical cancer (3).

It is well-known that minimal invasive surgery has advantages over open abdominal surgery. All comparisons between open and laparoscopic-based radical operations for the treatment of women with early cervical cancer favor laparoscopy with respect to blood loss, recovery, complication rates and cosmetic results (6-10). Laparoscopic operations result in identical oncological outcomes and have no adverse effect on overall prognosis and survival (6-10).

In this study, we aimed to establish and analyze TMMR using the laparoscopic approach, and to present our early results on operative morbidity and resection margins from 34 patients.

\section{Patients and Methods}

This study is a preliminary report of patients with cervical cancer of International Federation of Gynecology and Obstetrics (FIGO) 
stages IA-IIB, who were recruited between April 2012 and April 2016. They underwent laparoscopic TMMR as the primary treatment at the Charité, Virchow Campus, Berlin, Germany. Institutional Review Board approval was obtained under registration number EA1/174/14.

From April 2012, 34 patients with cervical cancer FIGO stages IA-IIB were treated by laparoscopic TMMR and total pelvic lymphadenectomy. Laparoscopic para-aortic lymphadenectomy was performed in cases of FIGO stage IIB and by positive sentinel lymph nodes from the common iliac region at frozen section.

All patients who underwent laparoscopic radical surgery were informed of the risks of the procedure (including conversion to laparotomy if necessary) and gave written informed consent. The same surgeon (MZM) performed all the laparoscopic interventions.

The inclusion criteria were body mass index (BMI) $\leq 35 \mathrm{~kg} / \mathrm{m}^{2}$, histopathological confirmed diagnosis of cervical carcinoma (via biopsy or conization), and no evidence of potential extra-pelvic disease (FIGO IA-IIB). Data were obtained retrospectively from medical and pathology records. The following criteria were analyzed: Tumor-related characteristics: Histological type, stage, TNM staging, tumor size and resection margins; operative criteria: Operative time, number of lymph nodes removed, intraoperative blood loss and complications; perioperative morbidity: Hemoglobin drop and postoperative complications (early and late).

Surgical technique. The senior author was trained in the technique for TMMR after participating in Professor Höckel's course at the Leipzig School of Surgery in 2011. Following his training he performed open TMMR at the Charité/Virchow Gynaecological Oncology Department for one year prior to commencing laparoscopic TMMR.

The principles of the surgical steps, described by Höckel et al. (11), were systematically translated to laparoscopic surgery to guarantee the same radicality whilst maintaining the advantages of minimally invasive surgery. From April 2012, all TMMR procedures were performed using the laparoscopic technique as follows.

Step 1: Surgical access is gained through five disposable 10/12 mm- trocars (ENDOPATH ${ }^{\circledR}$ XCEL $^{\mathrm{TM}}$ Bladeless Trocars, Ethicon, Somerville, NJ, USA). An Optic trocar is inserted umbilically, two lateral trocars about $2 \mathrm{~cm}$ above the anterior superior iliac spines bilaterally, a medial trocar $2 \mathrm{~cm}$ in the midline above the symphysis pubis, and a trocar at Palmer's point on the right $(3 \mathrm{~cm}$ below the right costal margin in the mid-clavicular line).

Step 2: The pelvic and mid-abdominal retroperitoneum is opened by incising the peritoneum at the psoas muscles, paracolic gutters, between the two leaves of the broad ligament and along the mesenteric root.

Step 3: The infundibulopelvic ligaments and ureters are exposed. The cecum, and sigmoid colon are mobilized. With the exception of the rectosigmoid colon, all bowel organs are retracted ventrally and placed in the right upper abdomen.

Step 4: Para-rectal spaces are developed with the hypogastric nerves adhering medially to the mesorectum. This maneuver is carried out down to the level where the pelvic splanchnic nerves join the hypogastric nerves to form the inferior hypogastric plexus but not further dorsally.

Step 5: The sigmoidal colon is elevated with the attached hypogastric plexus to the anterior abdominal wall. Lymph nodes in the common iliac region are dissected and examined under frozen section intraoperatively. Para-aortic lymph node dissection is performed in cases of FIGO stage IIB, or in any FIGO stage with positive nodes at frozen section. In cases of negative nodes, a systematic dissection of pelvic lymph nodes is performed. The parietal nodes of the anterior pelvic compartments (external, internal iliac and obturator) are removed by completely stripping the external and internal iliac artery and vein, exposing the proximal pelvic obturator nerves and the lumbar rami of the sacral plexus and removing the para-visceral pelvic fat pads. The obturator nerve, artery and vein, the arcus tendineus, and proximal sciatic nerve are also exposed.

Step 6: The round ligaments are transected. The anterior and posterior leaves of the broad ligaments are incised. If the adnexae are to be removed with the TMMR specimen, the infundibulopelvic ligaments are ligated and cut, otherwise the adnexae are separated from the uterus.

Step 7: Anteriorly, paravesical spaces are developed with the umbilical arteries adhering medially to the bladder, down to the pubococcygeus and iliococcygeus muscles, exposing the complete anterior side of the urogenital mesentery.

Step 8: The peritoneum of the vesico-uterine pouch is incised and the bladder is separated from the anterior cervix and the proximal vagina by dissection of the loose and dense sub-peritoneal connective tissue.

Step 9: The umbilical artery together with the superior bladder mesentery is separated from the anterior mesometrium.

Step 10: The uterine arteries and veins are coagulated and cut at their origins.

Step 11: The peritoneum of the recto-uterine pouch is incised and the anterior mesorectum is separated from the posterior vaginal wall down to the mid vagina. Laterally, the mesorectum is separated from the dense subperitoneal connective tissue ("uterosacral ligaments') to the level of the inferior hypogastric plexus.

Step 12: The proximal inferior hypogastric plexus is mobilized from the lateral surface of the dense sub-peritoneal connective tissue, which encases the rectum for $1-2 \mathrm{~cm}$ enabling the transection of the latter without nerve damage.

Step 13: Immediately above the superior margin of the inferior hypogastric plexus, the dense sub-peritoneal connective tissue ('rectouterine ligaments', 'uterosacral ligaments') is stepwise transected.

Step 14: The operation proceeds in the anterior compartment. The mesometrium is mobilized from its origin at the site of the already transected uterine artery and veins towards the uterus beyond the superior surface of the ureter.

Step 15: The vesicovaginal venous plexus together with the dense subperitoneal connective tissue above the pre-vesical segment of the ureter is coagulated and divided. After cutting the remaining dorsal attachments the ureter can be completely mobilized laterally.

Step 16: The vaginal wall is incised $2 \mathrm{~cm}$ beneath the cervical tumor thus completing the colpotomy. The TMMR specimen is removed through the vagina and the vaginal stump is closed with laparoscopical sutures.

Perioperatively, all patients received once-off intravenous antibiotic prophylaxis $(1.5 \mathrm{~g}$ cefuroxime plus $500 \mathrm{mg}$ metronidazole) at induction and low molecular weight heparin (0.4 $\mathrm{ml}$ Fraxiparin/24 h) subcutaneously $12 \mathrm{~h}$ before the operation. Intraoperative blood loss was estimated by subtracting the volume of the irrigation fluid from the total amount of fluid in the suction apparatus.

Endpoints of this study were status of the surgical margins, number of lymph nodes removed, short-term postoperative 
Table I. Postoperative complications graded according to the Franco-Italian Glossary (12).

\begin{tabular}{lccc}
\hline Organ system & Grade 1 & Grade 2 & Grade 3 \\
\hline Urinary & 3 (Urinary tract infection) & - & 1 (Cysto-vaginal fistula) \\
Cutaneous & 2 (Pressure sores) & - & - \\
Gastrointestinal & 1 (Paralytic Ileus) & - & - \\
Vascular & 1 (Deep vein thrombosis) & - & - \\
Pelvic soft tissue & - & 2 (Vaginal cuff dehiscence) & - \\
\hline
\end{tabular}

complications (within 30 days of the procedure), long-term postoperative complications (more than 30 days after the procedure), length of hospitalization, median follow-up duration, and recurrence rate.

\section{Results}

In total, 34 patients with FIGO stage IA-IIB cervical cancer underwent surgery. All patients underwent a laparoscopic TMMR. Therapeutic pelvic and parametric lymphadenectomy was added when indicated, as per national guidelines in Germany. The mean age of patients was 48.2 years (range $=30-74$ years).

Tumor-related characteristics. The group included 29 patients with squamous cell cancer $(85.3 \%)$, four patients with adenocarcinomas $(11.8 \%)$, and one case with a basaloid carcinoma $(2.9 \%)$. The mean tumor size was $25.6 \mathrm{~mm}$ (range $=3-55 \mathrm{~mm}$ ). Positive nodes were detected in $23.5 \%$ of cases ( $8 / 34$ patients). The frequency of high-grade tumors (G3) was $32.4 \%$ (11/34). Fifty percent (17/34) of tumors showed lymphangio invasion (L1). Hemangiosis carcinomatosa was diagnosed in only four out of 34 cases $(11.8 \%)$. A total of $35.3 \%(12 / 34)$ of patients received adjuvant radiochemotherapy with cisplatin following discussion at the multi-disciplinary tumor conference in spite of TMMR because it is still not the standard therapy in Germany. In one case, adjuvant radiochemotherapy was advised but the patient declined.

Operative criteria. All interventions were performed as intended and no transition to open surgery was necessary in any of the cases. Tumor resection was microscopically confirmed as R0 in 33/34 (97\%) cases, with one case showing microscopic residual tumor in the vagina. This patient underwent successful secondary vaginal vault resection. The mean extension of resected parametrium in our TMMR specimens was $43.8 \times 27.6$ (range $=30-75 \times 10-70)$ $\mathrm{mm}$ and $44.4 \times 30.8$ (range $=20-70 \times 15-70) \mathrm{mm}$ for the right and left sides, respectively.

Pelvic lymphadenectomy was performed in all cases, with a mean lymph node count of 43.7 (range=22-81). Para-aortic lymphadenectomy was performed in one case of FIGO stage
IIB. The ovaries were removed in three out of 34 patients. In the other cases, lateral ovaropexy was performed and the ovaries transposed just above the pelvic brim in the paracolic gutters.

The mean operative time for performing laparoscopic TMMR with laparoscopic pelvic lymph node dissection was 239 (range=120-540) $\mathrm{min}$. The mean intraoperative blood loss was only $65.2 \mathrm{ml}$ (range $=20-150 \mathrm{ml}$ ) with a mean hemoglobin drop of $2.9 \mathrm{~g} / \mathrm{dl}$ (range=1.8-4.6 g/dl). No blood transfusions were required intra- or postoperatively.

Intraoperative complications occurred in 4/34 (11.8\%) cases (two ureteric and two bladder injuries). All injuries were treated successfully laparoscopically in the same session.

Early perioperative morbidity. Postoperative complications occurred in 10/34 (29.4\%) patients and were graded using the Franco-Italian Glossary (Table I).

One intraoperative incidental cystotomy occurred at the time of laparoscopic bladder dissection of the anterior vagina. This was repaired as mentioned. Unfortunately, this resulted in the formation of a vesico-vaginal fistula which necessitated a second vaginal operation using Latzko's technique. This was the only case of a late postoperative complication.

The average hospital stay exceeded one week in most cases (range=3-20 days). However, the length of stay in our Department is related to our local discharge protocols and not to clinical necessities. Therefore, it is irrelevant to the scope of our study. The short follow-up showed only two recurrences, locoregional recurrence in the vaginal vault and peritoneal carcinosis. Both patients underwent a second operation for recurrent disease. However, the pathologist reviewed the histological study in the second case after receiving the new material from peritoneal carcinosis and changed it from adenocarcinoma of the cervix uteri to adenocarcinoma of the corpus uteri.

\section{Discussion}

In this study, we reported our initial experience with laparoscopic TMMR. In the past, several studies have shown 
the effectiveness and safety of open surgical TMMR. This was mainly reported in 2009 by Höckel et al. (3) in a prospective study of 212 patients over a 10-year period (without adjuvant therapy). The study reported that the surgical goal of resecting the Müllerian compartment was successful in all patients. Disease recurrence in the pelvis was only observed in $1.4 \%$ of patients, all of whom underwent successful secondary treatment (3).

Moreover, in patients with FIGO stage IB-IIA tumor, disease-free and overall survival rates were $98 \%$ in both. Patients with node-positive disease had a 5-year overall and disease-free survival rate of $91 \%$ and $81 \%$, respectively. In addition, the complication rates after a median of 41 months remained low, with $9 \%$ of patients having a grade 2 complication and $35 \%$ of patients reporting grade 1 complications [classified according to the Franco-Italian Glossary (12)].

There are very few studies reporting a minimally invasive approach to TMMR. In this preliminary report, we showed that TMMR, as described by Höckel et al., can be systematically translated to a laparoscopic approach. An obvious limitation to this study is the small sample size and short follow-up period. However, only one locoregional recurrence was recorded out of 34 cases $(2.9 \%)$ and the postoperative complication rates remained relatively low compared to open techniques of radical hysterecomy (7-9), with excellent resection margins.

Our findings support our previously published data from a multicentric study (13) with 71 patients admitted between July 2013 to August 2014 with early cervical cancer who underwent laparoscopic TMMR at three centers in Italy and Germany. Similar to our current findings, the mean operative time was 260 minutes, with minimal blood loss, and a relatively low intraoperative complication rate. The median follow-up time was shorter than in our study (18 months) and only two $(2.8 \%)$ early locoregional recurrences were observed. The study stressed the relatively low-grade postoperative autonomic urinary complications, which were attributed to the laparoscopic TMMR technique sparing the pelvic nerves supplying the bladder.

Moreover, another study by Kimmig et al. presented feasibility data of TMMR translated to robotic surgery (2). Twenty-six patients with FIGO stage IA-IIB cervical cancer were evaluated following treatment by robotic TMMR with robotic lymphadenectomy. The study reported no intraoperative complications and no transition to open surgery. The postoperative complication rate was $23 \%$. This is similar to our findings using the laparoscopic approach. There were no locoregional occurrences in the 18-month follow-up time. The study showed that TMMR can be effectively and safely translated to robotic surgery, adding the advantage of minimally invasive surgery.

Our study demonstrates that laparoscopic TMMR continues to provide the advantages of minimally invasive surgery and appears feasible and safe in treating patients with FIGO stage I-II cervical cancer. In the patients evaluated, no conversion to open surgery was necessary and none of the patients needed intraoperative or postoperative blood transfusion. Postoperative complications rates remained low.

It should be borne in mind that data were collected during the initial attempts at the method and that there is a learning curve, which we already described in a previous article (14). In addition, sample size is extremely small and there has as yet been no significant follow-up period. Consequently, we are aiming to increase the sample size and continue to follow-up patients in order to evaluate recurrence rates and late complications. Further large-scale prospective studies are needed to evaluate minimally invasive surgical techniques (laparoscopic and robotic) for the treatment of cervical cancer confined to the Müllerian compartment.

\section{Conflicts of Interest}

No financial or personal conflict of interest by any of the authors to declare.

\section{References}

1 Torre LA, Bray F, Siegel RL, Ferlay J, Lortet-Tieulent J and Jemal A: Global cancer statistics, 2012. CA Cancer J Clin 65(2): 87-108, 2015.

2 Kimmig R, Wimberger P, Buderath P, Aktas B, Iannaccone A and Heubner $\mathrm{M}$ : Definition of compartment-based radical surgery in uterine cancer: radical hysterectomy in cervical cancer as 'total mesometrial resection (TMMR) by M Höckel translated to robotic surgery (rTMMR). World J Surg Oncol 26(11): 211, 2013.

3 Höckel M, Horn LC, Manthey N, Braumann UD, Wolf U, Teichmann G, Frauenschläger K, Dornhöfer N and Einenkel J: Resection of the embryologically defined uterovaginal (Müllerian) compartment and pelvic control in patients with cervical cancer: a prospective analysis. Lancet Oncol 10(7): 683692, 2009.

4 Höckel M: Cancer permeates locally within ontogenetic compartments: clinical evidence and implications for cancer surgery. Future Oncol 8(1): 29-36, 2012.

5 Manjunath AP and Girija S. Embryologically based resection of cervical cancers: a new concept of surgical radicality. J Obstet Gynaecol India 62(1): 5-14, 2012.

6 Park CT, Lim KT, Chung HW, Lee KH, Seong SJ, Shim JU and Kim TJ: Clinical evaluation of laparoscopic-assisted radical vaginal hysterectomy with pelvic and/or paraaortic lymphadenectomy. J Am Assoc Gynecol Laparosc 9(1): 49-53, 2002.

7 Frumovitz M, dos Reis R, Sun CC, Milam MR, Bevers MW, Brown J, Slomovitz BM and Ramirez PT: Comparison of total laparoscopic and abdominal radical hysterectomy for patients with early-stage cervical cancer. Obstet Gynecol 110(1): 96-102, 2007.

8 Ghezzi F, Cromi A, Ciravolo G, Volpi E, Uccella S, Rampinelli $\mathrm{F}$ and Bergamini V: Surgicopathologic outcome of laparoscopic 
versus open radical hysterectomy. Gynecol Oncol 106(3): 502506, 2007.

9 Pomel C, Canis M, Mage G, Dauplat J, Le Bouedec G, Raiga J, Pouly JL, Wattiez A, Bruhat MA: Laparoscopically extended hysterectomy for cervix cancer: technique, indications and results. Apropos of a series of 41 cases in Clermont. Chirurgie 122(2): 133-136, 1997.

10 Krause N, Schneider A: Laparoscopic radical hysterectomy with paraaortic and pelvic lymphadenectomy. Zentralbl Gynakol 117: 346-348, 1995 (in German).

11 Höckel M, Horn LC, Hentschel B, Höckel S and Naumann G: Total mesometrial resection: High-resolution nerve-sparing radical hysterectomy based on developmentally defined surgical anatomy. Int J Gynecol Cancer 13(6): 791-803, 2003.

12 Chassagne D, Sismondi P, Horiot JC, Sinistrero G, Bey P, Zola P, Pernot M, Gerbaulet A, Kunkler I and Michel G: A glossary for reporting complications of treatment in gynecological cancers. Radiother Oncol 26(3): 195-202, 1993.
13 Chiantera V, Vizzielli G, Lucidi A, Gallotta V, Petrillo M, Legge F, Fagotti A, Sehouli J, Scambia G and Muallem MZ: Laparoscopic radical hysterectomy in cervical cancer as total mesometrial resection (L-TMMR): a multicentric experience. Gynecol Oncol 139(1): 47-51, 2015.

14 Muallem MZ, Feldheiser A and Sehouli J: Laparoscopic radical hysterectomy and lymph node dissection learning experience at campus Virchow - Charite Anticancer Res 33(8): 3449-3453, 2013.

Received June 18, 2016

Revised July 8, 2016

Accepted July 11, 2016 